\title{
Original
}

\section{Ultrastructural and Histopathological Analysis of Initial Change and Myoglobin Absorption on Proximal Tubule Injury with Glycerol-induced Rhabdomyolysis in Rats}

\author{
Tomomi Abe ${ }^{1)}$, Masahisa Inoue ${ }^{1)}$, Masumi Furutani' ${ }^{2)}$, Masahiko Akita ${ }^{1)}$ and Kojun Setsu ${ }^{1)}$ \\ 1) Laboratories for Structure and Function Research, Faculty of Pharmaceutical Sciences, Tokushima Bunri University, Yamashiro- \\ cho, Tokushima 770-8514, Japan. \\ 2) Central Research Laboratory, Okayama University Medical School, Shikata-cho, Okayama 700-8558, Japan. \\ (Accepted for publication, December 5, 2009)
}

\begin{abstract}
Rhabdomyolysis is a disorder of striped (skeletal) muscle and is caused by several factors, and acute renal failure associated with myoglobinuria is the most serious complication of rhabdomyolysis. However, the mechanism of tubule damage caused by myoglobin is still unclear. The early histopathological changes were not examined. Myoglobin reabsorption by the proximal renal tubules has not been reported to contribute to cellular damage. We performed histological analysis in a rat rhabdomyolysis model with administration of cytochalasin B (CB), which is an inhibitor of motile cell motile processes. CB inhibits the endocytosis of myoglobin in the proximal renal tubules and the increase in the BUN level, and decreased the percentage of area that had undergone tubular necrosis. On the other hand, initial degenerative changes (shortening of microvilli and protrusion of the cytoplasm) were observed on the cell surface of the proximal tubules with glycerolinduced cellular injury, and were not inhibited by the administration of CB. In conclusion, initial glycerolinduced cellular injury of the renal proximal tubules may be caused by free iron, which is isolated from myoglobin in urine. Further, the combination of the free iron in urine and the myoglobin reabsorbed by tubular cells may lead to cell necrosis.
\end{abstract}

Key words: Experimental rhabdomyolysis, Renal injury, Myoglobin, Cytochalasin B

\section{Introduction}

Rhabdomyolysis is a disorder of striped (skeletal) muscle and is caused by several factors, including trauma and the side effects of some kind of drugs. This condition results in considerable leakage of muscle-cell components, especially myoglobin, followed by myoglobinuria. Acute renal failure (ARF) associated with myoglobinuria is the most serious complication of rhabdomyolysis, and it may be fatal ${ }^{1-5)}$. Myoglobin is a heme protein, which contains iron; the oxidation of iron ions generates a hydroxyl radicals. These free hydroxyl radicals are believed to cause cellular injury in the proximal renal tubules ${ }^{1), 6), 7)}$. However, the mechanism of tubule damage caused by myoglobin is still unclear. Several authors have conducted histopathological analyses of myoglobin-induced renal injury by injecting glycerol in rats; however, in these studies, histopathological changes were analyzed more than 24 hours after glycerol injection ${ }^{8-12}$. The early histopathological changes were not examined. Furthermore, under

Correspondence to: Dr. Msahisa Inoue, Laboratories for Structure and Function Research, Faculty of Pharmaceutical Sciences, Tokushima Bunri University, Yamashiro-cho, Tokushima 770-8514, Japan, Tel: 088-6028474 Fax: 088-655-3051, E-mail: inoue@ph.bunri-u.ac.jp normal conditions, myoglobin is freely filtered by the glomerulus, enters the tubule epithelial cells through endocytosis, and is metabolized $^{1,2), 4)}$. Some studies suggest that free iron, which is isolated from myoglobin, is present in the urine and directly causes cellular damage ${ }^{1), 7)}$. Myoglobin reabsorption by the proximal renal tubules has not been reported to contribute to cellular damage.

Cytochalasin B (CB) and related drugs are well-known inhibitors of motile cell motile processes, including phagocytosis $^{13), 14)}$. The in vivo mechanism of action of cytochalasins remains unclear, although it is generally agreed that these drugs affect the organization of actin, which is thought to be responsible for cell motility ${ }^{13-16)}$. CB may inhibit the reabsorption of myoglobin in the proximal renal tubules.

To investigate the relationship between myoglobin reabsorption and cellular injury of proximal renal tubules caused by experimental rhabdomyolysis, we performed histological analysis in a rat rhabdomyolysis model by using immunohistochemistry, electron microscopy, and immunoelectron microscopy.

\section{Materials and Methods}

Animals 
J.Hard Tissue Biology Vol. 18(3):141-148, 2009

Male Wistar rats (weighing approximately. 160g) purchased from Japan SLC, Inc. (Shizuoka, Japan) were used in this study. They were housed under a standard light/dark cycle with free access to food (Type MF, Oriental Yeast, Japan) and water. The experimental protocols were approved by the animal ethics committee of Tokushima Bunri University.

\section{Grouping and drug administration}

We intramuscularly injected $10 \mathrm{mg} / \mathrm{kg}$ of $50 \%$ glycerol (Wako Pure Chemical Industries, LTD, Japan) in saline (Otsuka Pharmaceutical Co., Ltd. Tokushima, Japan) into the hind limbs of 6 rats (glycerol group; G group). Another 6 animals received an intraperitoneal injection of $150 \mu \mathrm{g} / \mathrm{kg}$ CB (from Drechslera dematioidea, Sigma Aldrich, Japan) just before the glycerol injection (glycerol + CB group; G + CB group). A third group of 6 animals were administered intraperitoneal and intramuscular injections of saline in a volume equivalent to that of glycerol and CB respectively (control group; C group).

\section{Biochemical analysis}

Twenty-four hours after the injections were administered, the animals were euthanized by administering an overdose of an anesthetic agent. Plasma separated from collected blood was assayed for blood urea nitrogen (BUN) by using the BUN B-test Wako (Wako Pure Chemical Industries, LTD, Japan). Statistical analysis was performed using analysis of variance (ANOVA) with the Student-Newman-Keuls test.

\section{Histological and morphological analysis}

At 6 or 24 hours after the injections were administered, the animals were euthanized by administering an overdose of an anesthetic agent. Their kidneys were removed and divided into 3 parts; each part was subjected to histological analysis as follows. A part of the isolated kidney was fixed with 10\% neutral buffered formalin. The specimens were embedded in paraffin blocks in a routine manner, and cut longitudinally into 4- $\mu \mathrm{m}$-thick sections for routine staining with hematoxylin-eosin (H.E.), periodic acidSchiff (PAS) reaction, or immunohistochemistry. The extent of tubular injury was evaluated in terms of the percentage of area showing a particular level of histological damage on HE staining. Statistically significant differences were determined by the Student's $t$ test.

\section{Immunohistochemistry}

For Immunohistochemical analysis, deparaffinized sections were immersed in $0.3 \%$ hydrogen peroxide in methanol to block endogenous peroxidase. Thereafter the sections were covered with anti-myoglobin antibody (Novocastra Laboratories Ltd., USA) and incubated overnight at $4^{\circ} \mathrm{C}$. The immunoreaction was performed using a Vectastain peroxidase ABC kit (Vector Laboratories, USA). The antigenic sites were identified after

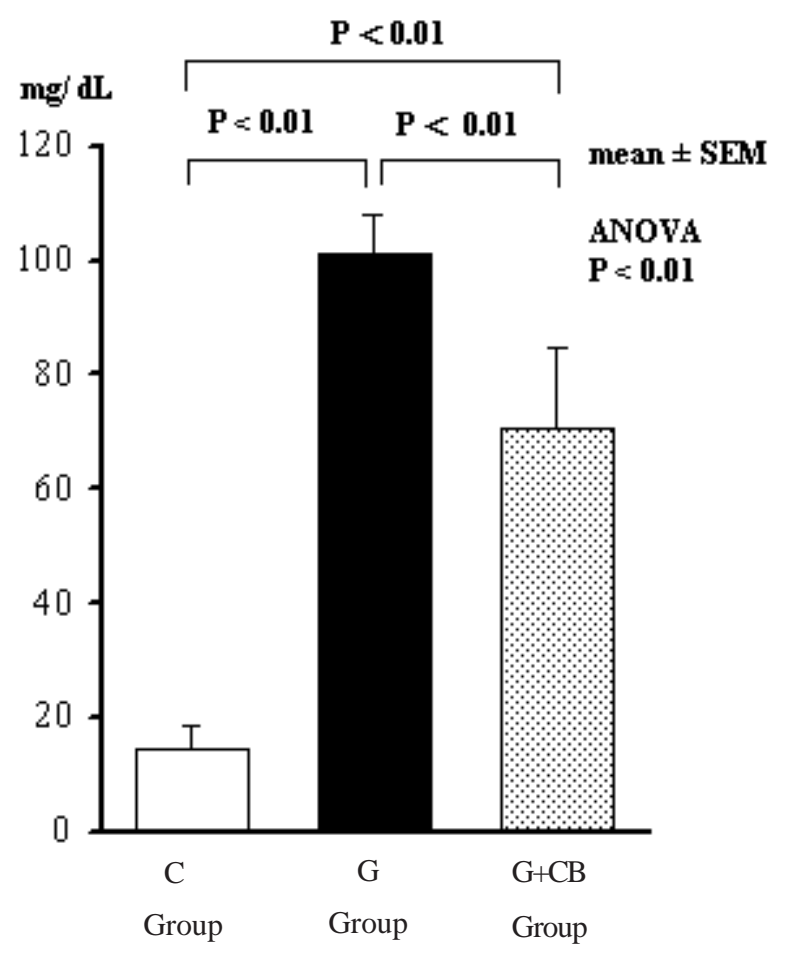

Fig. 1 Blood Urea Nitrogen (BUN) at 24 hours after the administration of injection. The values are expressed as mean \pm SEM. BUN level in $G$ group is significantly higher than that in $\mathrm{C}$ group $(\mathrm{p}<0.01)$. Administration of CB significantly inhibits the increase in BUN level as compared with G group ( $<$ 0.01). (ANOVA with Student-Newman-Keuls test)

reaction with $0.05 \%$ 3'3- diaminobenzidine hydrochloride (DAB; Dojindo, Japan) in $0.05 \mathrm{M}$ Tris buffer. The nuclei were counterstained with Mayer's hematoxylin (Merck, Germany).

\section{Electron microscopy}

Chopped pieces of kidneys, which were isolated 6 hours after the administration of the injections, were fixed with a freshly prepared solution of $2 \%$ paraformaldehyde and $2 \%$ glutaraldehyde in $0.1 \mathrm{M}$ phosphate buffer. These pieces were washed in $0.1 \mathrm{M}$ phosphate buffer and post-fixed with $1 \%$ buffered osmium tetroxide for 1-2 hours. The samples were dehydrated in increasing concentrations of ethanol, infiltrated in propylene oxide, and embedded in Epon 812 (TAAB Laboratories Equipment Ltd, England) at $60^{\circ} \mathrm{C}$ for 48 hours. They were cut into 80 -nm slices and stained with $2 \%$ uranyl acetate and lead citrate. Specimens were then observed under a transmission electron microscope $(\mathrm{H}-$ 7500; Hitachi, Japan) at $80 \mathrm{kV}$.

\section{Immunoelectron microscopy}

Chopped pieces of kidneys, which were isolated 6 hours after the administration of the injections, were fixed with a freshly prepared solution of $4 \%$ paraformaldehyde and $0.075 \%$ glutaraldehyde in $0.1 \mathrm{M}$ phosphate buffer. The samples were embedded in LR-White resin without postfixation. Ultrathin 
Tomomi Abe et al.: Myoglobin Absorption on Glycerol-induced Renal Injury
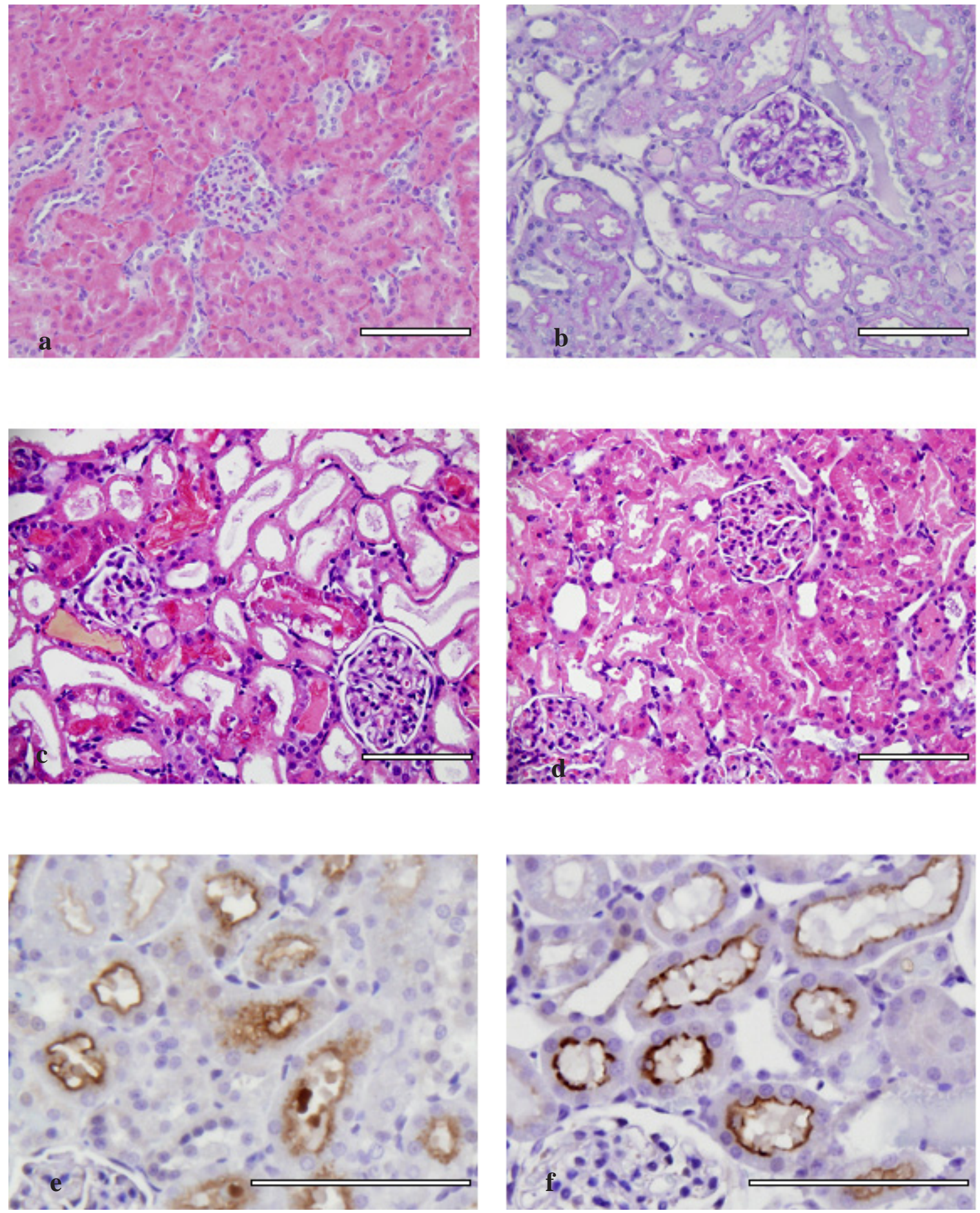

Fig. 2-a Light micrograph of kidney in C group at 24 hours showing normal architecture. (H.E. staining: Bar $=100 \mu \mathrm{m}$.)

Fig. 2-b Light micrograph of kidney in G group at 6 hours after glycerol injection showing cast formation in the distal tubules and shortening of brush border of proximal tubules. (PAS reaction: $\mathrm{Bar}=100 \mu \mathrm{m}$.)

Fig. 2-c Light micrograph of kidney in G group at 24 hours after glycerol injection showing cast formation and severe necrosis of proximal tubules. (H.E. staining: $\mathrm{Bar}=100 \mu \mathrm{m}$.)

Fig. 2-d Light micrograph of kidney in G + CB group at 24 hours after glycerol injection showing cast formation and degeneration of proximal tubules. Degeneration of proximal tubules is less severe than that in G group. (H.E. staining: Bar $=100 \mu \mathrm{m}$.)

Fig. 2-e Immunohistochemistry against myoglobin in G group at 6 hours after glycerol injection. Immunoreactivity for myoglobin was observed in the cytoplasm of epithelial cells of proximal tubules. (Bar $=100 \mu \mathrm{m}$.

Fig. 2-f Immunohistochemistry against myoglobin in G + CB group at 6 hours after glycerol injection. Immunoreactivity for myoglobin is localized only on the surface of proximal tubules. (Bar $=100 \mu \mathrm{m}$.) 
sections were hybridized with anti-myoglobin antibody (Novocastra Laboratories Ltd., USA) and probed with secondary anti-rabbit immunoglobulin (Ig)G and 10-nm immunogold. The sections were stained with uranyl acetate and lead citrate. The specimens were then observed under a transmission electron microscope (H-7500; Hitachi, Japan) at $80 \mathrm{kV}$.

\section{Results}

\section{BUN level as a renal function parameter}

The BUN level is known to be a renal-function parameter. Twenty-four hours after the administration of injections, the mean (SD) BUN level in the G group (101.0 mg/dL [7.09 mg/dL]) was significantly higher than that in the $\mathrm{C}$ group $(14.5 \mathrm{mg} / \mathrm{dL}[4.20$ $\mathrm{mg} / \mathrm{dL}])$. The administration of CB significantly inhibited the increase in the BUN level (70.3 mg/dL [14.5 mg/dL]) compared with the level in the G group (Fig. 1).

\section{Histological and morphological analysis}

The $\mathrm{C}$ group did not show any histological changes (Fig. 2-a). In the $\mathrm{G}$ group, 6 hours after glycerol injection, cast formation, a typical histological abnormality, was observed mainly in the medullar segment of the distal tubules and Henle's loops. Although proximal tubule necrosis was not observed at this time, shortening of the brush border was observed in the cortical segments of the proximal tubules (Fig. 2-b). In the G group, 24 hours after glycerol injection, necrosis of the tubular epithelium was most severe in the cortical segments of the proximal tubules (Fig. 2-c). In the G + CB group, 6 hours after glycerol injection, cast formation and shortening of the brush border were as severe as those in the $G$ group. In the $\mathrm{G}+\mathrm{CB}$ group, 24 hours after the administration of injections, degenerative changes were observed in the proximal tubules, but necrosis was not very severe (Fig. 2-d). In all the groups, glomerular damage was not prominent.

Morphological analysis showed that the percentage of area with tubular necrosis in the G + CB group (54.9\% [7.74\%]) was significantly lower than that in the C group (89.2\% [3.85\%]) at 24 hours after glycerol injection (Fig. 3).

\section{Immunohistochemistry}

In the $\mathrm{C}$ group, no immunoreactivity toward myoglobin was observed. In the G group, immunoreactivity toward myoglobin was observed in the cytoplasm of the proximal tubular cells and in the casts at 6 hours (Fig. 2-e). In contrast, in the G + CB group, immunoreactivity toward myoglobin was localized at the surface of the proximal tubular cells and not in the cytoplasm of these cells at 6 hours (Fig. 2-f ).

\section{Electron microscopic findings}

The $\mathrm{C}$ group did not show any ultrastructural changes. In the G group, 6 hours after glycerol injection, swelling and shortening of the brush border were observed in the proximal tubular cells.

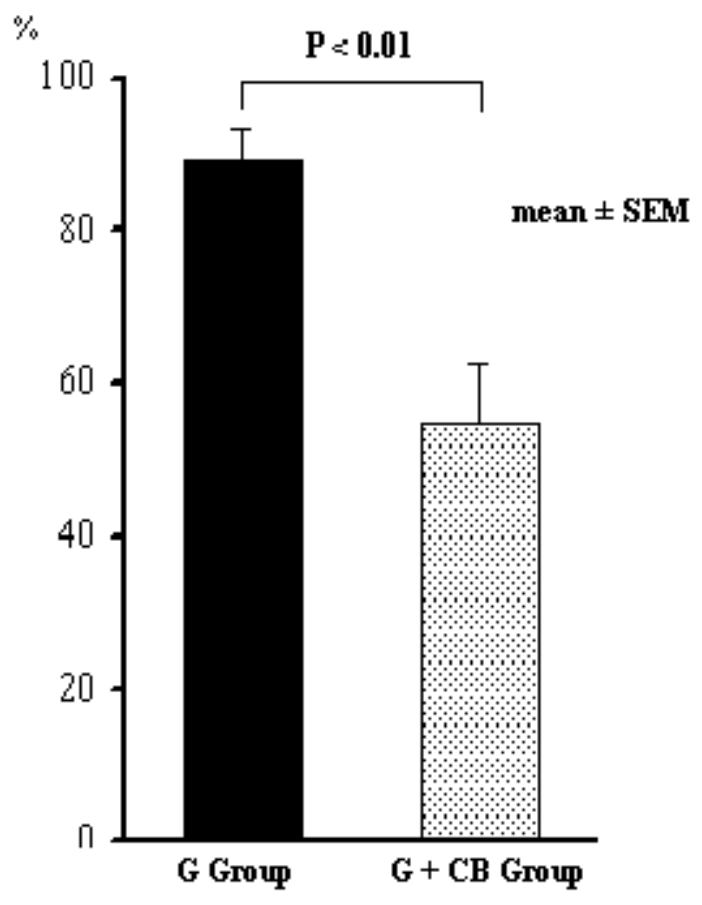

Fig. 3 Area ratio of tubular necrosis at 24 hours after the administration of injection. The values are expressed as mean \pm SEM. Area ratio of tubular necrosis in $\mathrm{G}+\mathrm{CB}$ group $(54.9+7.74 \%)$ is significantly lower than that in $\mathrm{C}$ group $(89.2+3.85 \%)(p<0.01)$ (Student's t test).

Numerous highly electron-dense bodies with the characteristic properties of lysosomes or endosomes were observed from the apical to the middle cytoplasm. However, lysosome-centric degeneration was not observed (Fig. 4-a, b). In some parts of the proximal tubular cells, microvilli had disappeared almost completely, and the plasma membrane protruded into the tubular lumen (Fig. 4-b). No degenerative changes were observed in the other organelles at this time. In the G + CB group, 6 hours after glycerol injection, shortening of microvilli and protrusion of the cytoplasm were observed; these changes were similar to those seen in the G group. However, the number of highly electrondense bodies was less than that in the G group, and these electrondense bodies were localized only in the apical cytoplasm. (Fig. 4c, d).

\section{Immunoelectron microscopic findings}

In the G group, 6 hours after glycerol injection, immunogold particles were observed predominantly in the electron-dense bodies, which exhibited the characteristic properties of lysosomes or endosomes; these particles were also localized in the cytoplasm or microvilli of proximal tubular cells (Fig. 4-e). In the G + CB group, 6 hours after glycerol injection, although immunogold particles were observed in the cytoplasm and brush border of the proximal tubular cells, these particles had not been accumulated in the lysosomes (Fig. 4-f). 
Tomomi Abe et al.: Myoglobin Absorption on Glycerol-induced Renal Injury
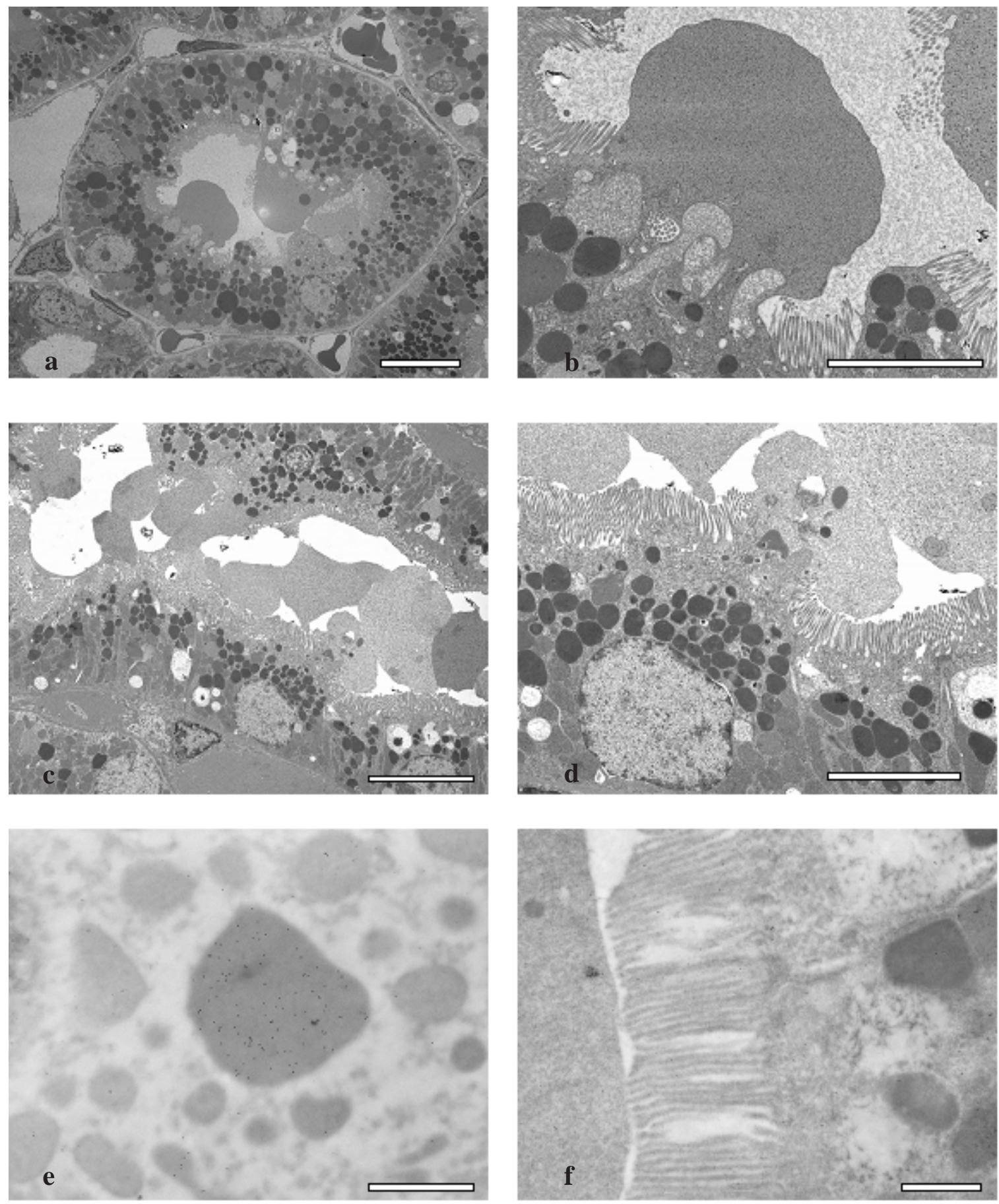

Fig. 4-a Electron micrograph of the renal proximal tubular cell in G group at 6 hours after glycerol injection showing shortening of the brush border, and numerous highly electron-dense bodies with the characteristic properties of lysosomes from the apical to the middle cytoplasm. (Bar $=10 \mu \mathrm{m}$ )

Fig. 4-b Electron micrograph of the renal proximal tubular cell in $\mathrm{G}$ group at 6 hours after glycerol injection. Microvilli had disappeared almost completely, and the plasma membrane protruded into the tubular lumen. $(\mathrm{Bar}=5 \mu \mathrm{m})$

Fig. 4-c Electron micrograph of the renal proximal tubular cell in G + CB group at 6 hours after glycerol injection. Shortening of microvilli were observed. The number of highly electron-dense bodies was less than that in the G group, and these were localized only in the apical cytoplasm. (Bar $=10 \mu \mathrm{m})$

Fig. 4-d Electron micrograph of the renal proximal tubular cell in G + CB group at 6 hours after glycerol injection. Shortening of microvilli and protrusion of the cytoplasm were observed. (Bar $=5 \mu \mathrm{m}$ )

Fig. 4-e Immunoelectron micrograph against myoglobin in G group at 6 hours after glycerol injection. Immunogold particles were observed predominantly in the electron-dense bodies. (Bar $=1 \mu \mathrm{m})$

Fig. 4-f Immunoelectron micrograph against myoglobin in G + GB group at 6 hours after glycerol injection. Immunogold particles had not been accumulated in the electron-dense bodies. $(\mathrm{Bar}=1 \mu \mathrm{m})$ 


\section{Discussion}

Acute kidney injury associated with myoglobinuria is the most serious complication of rhabdomyolysis and may be life threatening ${ }^{1), 2)}$. Rhabdomyolysis-induced renal injury is considered to be caused by a combination of renal vasoconstriction, myoglobin-associated oxidative injury, and cast formation ${ }^{1), 5), 17)}$. Glycerol injection is the most widely used model for experimental rhabdomyolysis-induced renal injury ${ }^{8), 18)}$. Several authors have conducted histopathological analyses of myoglobininduced renal injury by injecting glycerol in rats; however, in these studies, histopathological changes were analyzed more than 24 hours after glycerol injection, and early histopathological changes were not examined ${ }^{8-11)}$. In the present study, early changes during the development of glycerol-induced renal injury, i.e., within 6 hours after glycerol injection, were assessed using light and electron microscopy. Six hours after glycerol injection, shortening of the brush border was observed on both light and electron microscopy, and protruding cytoplasm was observed on electron microscopy. These findings may represent initial degenerative changes in the proximal tubules; these changes play a part of degenerative effects, which eventually lead to necrosis, and may be caused by myoglobin-associated oxidative injury.

Myoglobin is a heme protein; it contains iron, in the form of ferrous oxide $\left(\mathrm{Fe}^{2+}\right)$, which is necessary for binding molecular oxygen. However, molecular oxygen can promote the oxidation of $\mathrm{Fe}^{2+}$ to ferric oxide $\left(\mathrm{Fe}^{3+}\right)$, thus generating a hydroxyl radical, which causes cellular injury ${ }^{1), 2)}$. Heme and free iron-driven hydroxyl radicals are considered to be important mediators of tubule damage ${ }^{1), 7)}$. It is considered that at a urinary $\mathrm{pH}$ of $<5.6$, myoglobin dissociates into ferrihemate and globin. Ferrihemate contains $\mathrm{Fe}^{3+}$, and has a direct nephrotoxic effect, which potentiates renal tubular necrosis ${ }^{4), 5)}$. In contrast, under normal conditions, myoglobin is freely filtered by the glomerulus, enters the tubule epithelial cells through endocytosis, and is metabolized ${ }^{1)}$. However, myoglobin endocytosis has not been reported to be involved in rhabdomyolysis-induced renal injury. In the present study, we used CB to investigate the involvement of myoglobin endocytosis in glycerol-induced renal injury. CB is a well-known inhibitor of motile cell processes, including particle phagocytosis ${ }^{13), 14)}$. The in vivo mechanism of action of cytochalasins remains unclear, although it is generally agreed that these drugs affect the organization of the actin-containing microfilament system, which is thought to be responsible for cell motility ${ }^{13-16)}$. CB effectively inhibits phagocytosis in a variety of mammalian cell types in a rapidly reversible manner ${ }^{13), 14)}$. In the present study, immunoelectron microscopy showed that immunogold particles that reacted with myoglobin were present in the lysosomes of the proximal renal tubules at 6 hours after glycerol injection. Further, the accumulation of immunogold particles in the lysosomes was suppressed by the administration of $\mathrm{CB}$. This suggests that $\mathrm{CB}$ inhibits the endocytosis of myoglobin in the proximal renal tubules. In the present study, the administration of CB significantly inhibited the increase in the BUN level and decreased the percentage of area that had undergone tubular necrosis at 24 hours after glycerol injection. These results suggest that endocytosis of myoglobin may contribute to renal cytotoxicity. Recently, it has been shown that myoglobin exhibits peroxidase-like enzyme activity that leads to uncontrolled oxidation of biomolecules and lipid peroxidation ${ }^{1)}$, 19). This enzymatic activity of myoglobin, and not of the free iron isolated from myoglobin, may play an important role in the development of cellular injury mediated by absorbed myoglobin. Furthermore, initial degenerative changes (shortening of microvilli and protrusion of the cytoplasm) were observed on the cell surface and were not inhibited by the administration of CB. These results suggest that these degenerative changes may result from the free iron isolated from myoglobin and may not be closely related to the endocytosis of myoglobin by the proximal renal tubules.

In conclusion, initial glycerol-induced cellular injury of the renal proximal tubules is caused by free iron, which is isolated from myoglobin in urine. Further, the combination of the free iron in urine and the myoglobin reabsorbed by tubular cells may lead to cell necrosis.

\section{References}

1. Bosch X, Poch E, and Grau JM. Rhabdomyolysis and Acute Kidney Injury. N Engl J Med 361: 62-72, 2009

2. Vanholder R, Sever MS, Erek E, and Lameire N. Rhaabdomyolysis. J Am Soc Nephrol. 11(8): 1553-61, 2000

3. Poels PJ, and Gabreëls FJ. Rhabdomyolysis: a review of the literature. Clin Neurol Neurosurg. 95(3): 175-92, 1993

4. Khan FY. Rhabdomyolysis: a review of the literature. Neth J Med. 67(9): 272-83, 2009

5. Zager RA. Rhabdomyolysis and myohemoglobinuric acute renal failure. Kidney International. 49: 314-326, 1996

6. Scott JA, Fischman AJ, Homcy CJ, Fallon JT, Khaw BA, Peto CA, Rabito CA. Morphologic and functional correlates of plasma membrane injury during oxidant exposure. Free Radic Biol Med. 6(4): 361-7, 1989

7. Zager RA, and Foerder CA. Effects of inorganic iron and myoglobin on in vitro proximal tubular lipid peroxidation and cytotoxicity. J Clin Invest. 89: 989-95, 1992

8. Chander V, Singh D, and Chopra K, Catechin, a natural antioxidant protects against rhabdomyolysis-induced myoglobinuric acute renal failure. Pharmacol Res. 48(5): 503-9, 2003

9. Lochhead KM, Kharasch ED, and Zager RA. Anesthetic effects on the glycerol model of rhabdomyolysis-induced acute renal failure in rats. J Am Soc Nephrol. 9(2): 305-9, 1998 
Tomomi Abe et al.: Myoglobin Absorption on Glycerol-induced Renal Injury

10. Ustundag S, Yalcin O, Sen S, Cukur Z, Ciftci S, Demirkan

B. Experimental myoglobinuric acute renal failure: the effect of vitamin C. Ren Fail. 30(7): 727-35, 2008

11. Shah SV, and Walker PD. Evidence suggesting a role for hydroxyl radical in glycerol-induced acute renal failure. Am J Physiol. 255: F438-43, 1988

12. Chander V, Singh D, and Chopra K. Attenuation of glycerolinduced acute renal failure in rats by trimetazidine and deferoxamine. Pharmacology. 67(1): 41-48, 2003

13. Painter RG, Whisenand J, and McIntosh AT. Effects of cytochalasin B on actin and myosin association with particle binding sites in mouse macrophages: implications with regard to the mechanism of action of the cytochalasins. J Cell Biol. 91: 373-84, 1981

14. Axline SG, and Reaven EP. Inhibition of phagocytosis and plasma membrane mobility of the cultivated macrophage by cytochalasin B. Role of subplasmalemmal microfilaments.
J Cell Biol. 62(3): 647-59, 1974

15. Davies P, and Allison AC. Effects of cytochalasin B on endocytosis and exocytosis. Front Biol. 46: 143-60, 1978

16. Ohta M, Marceau N, and French SW. Changes in the organization and antigenic determinants of intermediate filaments of rat hepatocytes after infusion of cytochalasin B in vivo. Am J Pathol. 133(3): 578-88, 1988

17. Reineck HJ, O’Connor GJ, Lifschitz MD, and Stein JH. Sequential studies on the pathophysiology of glycerolinduced acute renal failure. J Lab Clin. 96(2): 356-62, 1980

18. Brandao R, Acker CI, Leite MR, Barbosa NB, and Nogueira CW. Diphenyl diselenide protects against glycerol-induced renal damage in rats. J Appl Toxicol. 29(7): 612-8, 2009

19. Reeder BJ, and Wilson MT. Hemoglobin and myoglobin associated oxidative stress: from molecular mechanisms to disease states. Curr Med Chem 12: 2741-51, 2005 
J.Hard Tissue Biology Vol. 18(3):141-148, 2009 